\title{
Síntesis, Caracterización y Evaluación Fotocatalítica de Puntos cuánticos de CdSe cubiertos con 2 tipos de Tioles
}

\author{
Manuel A. Triana ${ }^{(1)^{*}}$, Andrés F. López ${ }^{(1)(2)}$ y Rubén J. Camargo ${ }^{(1)}$ \\ (1) Escuela de Ingeniería Química, Universidad del Valle, Ciudad Universitaria Meléndez, A. A. 25360, \\ Cali-Colombia (e-mail: manuel.triana@correounivalle.edu.co; \\ andres.lopez.vasquez@correounivalle.edu.co; \\ ruben.camargo@correounivalle.edu.co) \\ (2) Programa de Ingeniería Ambiental, Universidad Libre Sede Bosque, Edificio Ingeniería, Oficina 208, \\ Bogotá-Colombia
}

Recibido Ene. 5, 2015; Aceptado Mar. 11, 2015; Versión final Mar. 20, 2015, Publicado Oct. 2015

\begin{abstract}
Resumen
En este artículo se presenta la síntesis acuosa de puntos cuánticos (PCs) de seleniuro de cadmio (CdSe) con dos tipos de agentes envolventes: ácido tioglicólico (TGA) y ácido mercaptosuccínico (MSA). A partir de los espectros UV-vis se calcularon la energía de banda prohibida óptica $\left(\mathrm{E}_{\mathrm{g}}{ }^{\mathrm{op}}\right)$, el diámetro promedio de partícula, y se construyó la curva de crecimiento de los nanocristales. Por medio de los espectros de fotoluminiscencia se halló un ancho asociado a la distribución de tamaños (ATMM). Finalmente, se evaluó la fotolisis de $\mathrm{H}_{2} \mathrm{O}$ usando PCs suspendidos en solución acuosa $(50 \mathrm{mg}$ en $0.1 \mathrm{~L}$ ) con sulfito de sodio $\left(\mathrm{Na}_{2} \mathrm{SO}_{3}: 0.1 \mathrm{M}\right)$ como donante sacrificial de electrones. Los PCs con envolvente de MSA mostraron evolución de $\mathrm{H}_{2}$ limitada por difusión y una tasa máxima de $1.126 \mu \mathrm{mol} \mathrm{H}_{2} / \mathrm{h}$ para PCs de $2.1 \mathrm{~nm}$. En el caso de PCs con TGA no se presentó evolución de $\mathrm{H}_{2}$.
\end{abstract}

Palabras clave: síntesis de nanocristales, puntos cuánticos, fotoluminiscencia, semiconductor fotocatalizador, fotólisis del $\mathrm{H}_{2} \mathrm{O}$

\section{Synthesis, Characterization and Photocatalytic Evaluation of CdSe Quantum dots capped with 2 types of Thiols}

\begin{abstract}
This paper presents the aqueous synthesis of cadmium selenide quantum dots (CdSe QDs) with two types of capping agents: thioglycolic acid (TGA) and mercaptosuccinic acid (MSA). Optical band gap energy $\left(E_{g}{ }^{o p}\right)$, mean diameter of particle and growth curves of nanocrystals were obtained from the absorption spectra. The width associated with the size distribution of the nanoparticles (FWHM) was found using the photoluminescence spectra. Finally, photochemical water splitting was evaluated using suspended QDs in aqueous solution $\left(50 \mathrm{mg}\right.$ on $0.1 \mathrm{~L}$ ) and sodium sulfite $\left(\mathrm{Na}_{2} \mathrm{SO}_{3}: 0.1 \mathrm{M}\right)$ as sacrificial electron donor. The QDs capped with MSA showed $\mathrm{H}_{2}$ evolution limited by diffusion and a maximum rate of $1.126 \mu \mathrm{mol} \mathrm{H}_{2} / \mathrm{h}$ for QDs of $2.1 \mathrm{~nm}$. On the other hand, QDs capped with TGA did not show hydrogen evolution.
\end{abstract}

Keywords: synthesis of nanocrystals, quantum dots, photoluminescence, photocatalyst semiconductor, photochemical water splitting. 


\section{INTRODUCCIÓN}

Un material semiconductor comúnmente usado tanto en celdas fotovoltaicas como en fotocatálisis es el óxido de titanio $\left(\mathrm{TiO}_{2}\right)$, en forma de película o como nanopartículas en suspensión (Suaterna, Insignares, Mueses, y Camargo. 2012) (Mesa, Machuca y Mueses. 2012). Sin embargo debido a sus propiedades limitadas como banda prohibida ancha (anatasa: $3.2 \mathrm{eV}$ ) y alto índice de dispersión de la radiación solar, la investigación se ha extendido a la sensibilización del $\mathrm{TiO}_{2}$ y al uso de otros nanomateriales con propiedades ajustables. Una alternativa ampliamente investigada en la actualidad para sensibilizar y aumentar la eficiencia de celdas fotovoltaicas y fotoquímicas es el empleo de nanocristales semiconductores conocidos como puntos cuánticos (PCs). Los PCs obtenidos en medio acuoso tienen estabilidad a largo término, intervalo de absorción desde el UV al IR (gracias a su estructura de banda ajustable) y pueden exhibir multiplicación de portadores de carga (Nozik, 2002). En particular, comparados con PCs sintetizados en solventes orgánicos los PCs en medio acuoso constituyen una mejor opción por su menor costo de síntesis, mejor reproducibilidad y menor impacto ambiental (Gaponik et al, 2002) (Wang y Liu. 2012). PCs de distinta composición han sido ampliamente investigados debido a sus propiedades optoelectrónicas únicas, sin embargo, su aplicación en sistemas de conversión y su interacción con electrolitos y semiconductores sólidos continúa siendo tema de investigación. En especial, la investigación está enfocada en el mejoramiento de la eficiencia de los dispositivos de conversión solar maximizando tanto el aprovechamiento del espectro solar (incluyendo el infrarrojo) como la transferencia de carga en la superficie de los PCs.

Los PCs de seleniuro de cadmio (CdSe) ya se han probado tanto en celdas fotovoltaicas híbridas (Kniprath et al., 2009) como en fotocatálisis para producción de hidrógeno con resultados aceptables (Holmes et al., 2011). Zhao et al., (2013) confirmaron experimentalmente una relación directa entre el grado de confinamiento cuántico de PCs de CdSe cubiertos con 2-mercaptoetanol (MCE) y la fotólisis del agua en un sistema PC/electrolito. Sin embargo, la transferencia de carga en la interfase PC-electrolito de celdas fotoquímicas y en la interfase PC-semiconductor de celdas fotovoltaicas debe mejorarse empleando pasivación superficial de los PCs y agentes estabilizantes de cadena corta. En la síntesis de PCs coloidales el agente estabilizante, usualmente un tipo de molécula orgánica, forma una capa envolvente sobre la superficie de los PCs estabilizándolos en el solvente de síntesis. Los criterios para mejorar la eficiencia de los PCs variando las propiedades del agente envolvente deben aclararse.

El rol del material fotogenerador es el más importante y sus propiedades deben ser ajustadas para aumentar el aprovechamiento del espectro solar, reducir la recombinación de pares electrón-hueco y evitar la fotodegradación. Es por tanto, que en el presente artículo se investigaron propiedades de fotogeneración de los PCs de CdSe y el desempeño de la envolvente de los $\mathrm{PCs}$ en la fotolisis del $\mathrm{H}_{2} \mathrm{O}$. En primer lugar, se sintetizaron PCs de CdSe con dos envolventes diferentes (TGA y MSA) y se hicieron crecer sometiendo la solución cruda a un ciclo de evaporación-condensación también llamado periodo de reflujo. Mediante el monitoreo del pH en la reacción del precursor de $\mathrm{Se}^{-2}$ se halló un tiempo ideal de máxima concentración, lo cual mejoró la reproducibilidad y el rendimiento de producción de los PCs. Posteriormente se realizaron pruebas de caracterización de los PCs en solución y en polvo para determinar sus propiedades optoelectrónicas y morfológicas. Por último, los PCs sintetizados y caracterizados fueron ensayados como catalizadores en la fotolisis de $\mathrm{H}_{2} \mathrm{O}$ en un fotorreactor con vaso de inmersión y lámpara de xenón de $55 \mathrm{~W}$. El análisis del desempeño y las tasas de evolución permitió comparar la influencia de: los agentes envolventes, la temperatura de la solución PC/electrolito y el tamaño y banda prohibida de los PCs de CdSe.

\section{METODOLOGÍA}

La metodología se presenta en varias secciones para mayor claridad: reactivos y materiales, síntesis de PCs, crecimiento de los PCs por reflujo, obtención del polvo de PCs de CdSe, técnicas de caracterización y equipos, cálculo de la energía de banda prohibida óptica, evaluación fotocatalítica de los PCs y el montaje experimental de la fotólisis

\section{Reactivos y materiales}

Las marcas y la pureza de los reactivos utilizados en cada síntesis de los PCs de CdSe son: Cloruro de cadmio hemidihidratado $\mathrm{CdCl}_{2} 2,5 \mathrm{H}_{2} \mathrm{O}$ (Alfa Aesar, $80.7 \%$ ), Ácido tioglicólico TGA (Panreac, $80 \%$ ), Ácido mercaptosuccínico MSA (Sigma-Aldrich, 97\%), borohidruro de sodio $\mathrm{NaBH}_{4}$ (Panreac, $96 \%$ ) y selenito de sodio $\mathrm{Na}_{2} \mathrm{SeO}_{3}$ (Sigma $\sim 98 \%$ ).

\section{Síntesis de PCs de CdSe/MSA}

La preparación de PCs de CdSe cubiertos con MSA se basó en el procedimiento publicado por Wang et al., (2012) pero se aplicaron las siguientes modificaciones: 1) La obtención de la solución cruda de PCs (sin 
reflujo) se realizó en atmosfera inerte de $\mathrm{N}_{2}$ dentro de una cabina hermética (Glove box); 2) La razón molar $\mathrm{Cd} / \mathrm{MSA} / \mathrm{Se}$ empleada fue: 1/1.6/0.15; y 3) Se invirtió el orden de adición de los reactivos (primero $\mathrm{Na}_{2} \mathrm{SeO}_{3}$ ya que el $\mathrm{NaBH}_{4}$ reacciona lentamente con el agua). Adicionalmente se usó un tiempo ideal de la reacción de formación del $\mathrm{Se}^{-2}$.

Las cantidades de reactivos y condiciones utilizadas se describen a continuación. Para la preparación del precursor de $\mathrm{Cd}^{+2}$ se disolvieron $0.5659 \mathrm{~g}$ de $\mathrm{CdCl}_{2} .2,5 \mathrm{H}_{2} \mathrm{O}$ en $100 \mathrm{~mL}$ de agua tipo I dentro de un matraz (tres bocas) de $250 \mathrm{~mL}$. Manteniendo el matraz sobre una plancha de agitación se añadieron $0.4954 \mathrm{~g}$ de MSA y se ajustó el pH a 11.0 empleando una solución $0.1 \mathrm{M}$ de $\mathrm{NaOH}$. Para la preparación del precursor de $\mathrm{Se}^{-2}$ se disolvieron $0.053 \mathrm{~g}$ de $\mathrm{Na}_{2} \mathrm{SeO}_{3}$ en $5 \mathrm{~mL}$ de agua tipo I dentro de un tubo colorimétrico de $10 \mathrm{~mL}$. Mientras la solución de $\mathrm{Na}_{2} \mathrm{SeO}_{3}$ fue agitada (magneto miniatura) se añadieron $0.1 \mathrm{~g}$ de $\mathrm{NaBH}_{4}$. El tubo se mantuvo abierto para liberar el gas producido durante la reacción. Cuando se alcanzó un tiempo de reacción de 6.2 min (rojo parduzco intenso) la solución de Se $\mathrm{Se}^{-2}$ fue inyectada dentro de la solución de Cd y tiol. La reacción del $\mathrm{Cd}^{+2}$ y el $\mathrm{Se}^{-2}$ produce una solución con un tono entre amarillo y naranja brillante. Todo el proceso de síntesis anterior se llevó a cabo a temperatura ambiente y en atmósfera inerte de $\mathrm{N}_{2}$, inmediatamente después se realizó el montaje de reflujo para el crecimiento de los nanocristales (NCs) a $98{ }^{\circ} \mathrm{C}$ durante $8 \mathrm{~h}$.

\section{Síntesis de PCs de CdSe/TGA}

En el caso de los PCs de CdSe/TGA, se reprodujo el procedimiento reportado por Wang et al., (2010) aplicando modificaciones similares al caso anterior: 1) Igual que en el caso anterior se usó la reacción $\mathrm{NaBH}_{4}$ $+\mathrm{Na}_{2} \mathrm{SeO}_{3}$ para la obtención del precursor de $\mathrm{Se}^{-2}$, en lugar de la reacción $\mathrm{NaBH}_{4}+\mathrm{Se}$, detallada por Klayman y Griffin (1973); 2) La razón molar Cd/TGA/Se empleada fue: 1/3.45/0.6; 3) La obtención de la solución cruda de PCs (sin reflujo) se realizó en atmosfera inerte de $\mathrm{N}_{2}$; y 4) Se realizó un reflujo a $98{ }^{\circ} \mathrm{C}$ de los NCs durante $8 \mathrm{~h}$, en lugar de síntesis rápida a temperatura ambiente.

Las cantidades de reactivos y condiciones de la síntesis se describen a continuación. Para la preparación del precursor de $\mathrm{Cd}^{+2}$ se disolvieron $0.5659 \mathrm{~g}$ de $\mathrm{CdCl}_{2} .2,5 \mathrm{H}_{2} \mathrm{O}$ en $100 \mathrm{~mL}$ de agua tipo I dentro de un matraz (tres bocas) de $250 \mathrm{~mL}$. Luego, agitando continuamente se añadieron $0.625 \mathrm{~mL}$ de TGA y se ajustó el pH a 11.2 empleando una solución $0.1 \mathrm{M}$ de $\mathrm{NaOH}$. Para la preparación del precursor de $\mathrm{Se}^{-2}$ se disolvieron 0.106 g de $\mathrm{Na}_{2} \mathrm{SeO}_{3}$ en $10 \mathrm{~mL}$ de agua tipo I dentro de un tubo colorimétrico. Mientras se agitaba la solución de $\mathrm{Na}_{2} \mathrm{SeO}_{3}$ se añadieron $0.2 \mathrm{~g}$ de $\mathrm{NaBH}_{4}$. El tiempo ideal de reacción en este caso fue de $3 \mathrm{~min}$. De igual manera todo el proceso de síntesis anterior se llevó a cabo a temperatura ambiente y en atmósfera inerte de $\mathrm{N}_{2}$, inmediatamente después se realizó el montaje de reflujo para el crecimiento de los NCs a $98{ }^{\circ} \mathrm{C}$ durante $8 \mathrm{~h}$.

\section{Crecimiento de los PCs por reflujo}

Una vez terminada la 1ra etapa de la síntesis (nucleación), los NCs se hicieron crecer mediante un ciclo de evaporación-condensación a la temperatura de ebullición del solvente $\left(98^{\circ} \mathrm{C}\right.$ para el agua en la atmósfera de Cali Colombia). El montaje de la etapa de crecimiento es un sistema cerrado que consiste en poner el matraz de la solución de PCs sobre una plancha de calentamiento y conectar la boca central del matraz a un condensador con agua refrigerante. El conteo del tiempo se inició una vez alcanzada la temperatura de ebullición de la solución. Luego, durante el reflujo se tomó una muestra de $5 \mathrm{~mL}$ cada hora hasta completar un periodo de 8 horas. Para extraer las muestras se introdujo una jeringa de $9 \mathrm{~cm}$ en medio de una tapa septum ubicada en una de las bocas laterales del matraz.

\section{Obtención del polvo de PCs de CdSe}

Para realizar pruebas de fotocatálisis y mediciones de propiedades como tipo de cristal (DRX) y composición superficial (FTIR) se requiere que los PCs estén en estado sólido (polvo), por lo cual, estos fueron precipitados desde la solución original empleando un anti-solvente polar (Gaponik et al. 2002).

El procedimiento de obtención de los NCs en polvo consistió en los siguientes pasos. Se tomó un volumen de la solución resultante del periodo de reflujo de $8 \mathrm{~h}$. Luego la solución fue concentrada 5 veces usando un evaporador rotatorio al vacío, es decir, en el caso de $50 \mathrm{~mL}$ de solución de PCs se evaporaron $40 \mathrm{~mL}$ de agua a una temperatura de ebullición $<98^{\circ} \mathrm{C}\left(70-80^{\circ} \mathrm{C}\right)$. A continuación, la solución concentrada se puso bajo agitación y se añadieron gotas de 2-propanol hasta que la solución se tornó bastante turbia. La solución turbia se dejó agitar otros 15 minutos y posteriormente se centrifugó por un periodo de 30 min. Con las fases sólida y líquida visiblemente separadas en los tubos de centrifugado, se procedió a extraer el agua sobrenadante por medio de una jeringa. Finalmente, las muestras húmedas fueron introducidas en un horno de secado durante $1.5 \mathrm{~h}$ y bajo una temperatura de $80^{\circ} \mathrm{C}$. El tiempo de secado dependió del espesor de la capa sólida precipitada. 


\section{Técnicas de caracterización y equipos}

Para medir las propiedades ópticas y morfológicas de los PCs se usaron las siguientes técnicas de caracterización y equipos: 1) Espectro de absorción UV-vis medido en un Spectroquant Pharo 300 de Merck. Cada muestra se introdujo en una cubeta con longitud de $10 \mathrm{~mm}$, y el espectro se midió en un intervalo de $300-700 \mathrm{~nm}$; 2) Espectro de fluorescencia o de fotoluminiscencia (PL) medido en un espectrofluorímetro Jasco FP-8500. La medición se realizó bajo una longitud de onda de excitación de $365 \mathrm{~nm}$. En general, fue necesario configurar el detector en "sensibilidad baja" y utilizar soluciones muy diluidas para evitar que la intensidad medida se saliera de rango (>10000). Dado que la dilución de las muestras fue cualitativa (2 gotas en $~ 3 \mathrm{~mL}$ ), los espectros de fluorescencia de las muestras no se pueden comparar correctamente en intensidad. Por tanto, los espectros PL se normalizaron y los datos relevantes extraídos fueron la longitud de onda de emisión y el ancho total a la mitad del máximo (ATMM); 3) Espectro infrarrojo por transformada de Fourier tomado en un Jasco FT/IR-4100. En cada medición se utilizó el polvo de los PCs puro y macerado, y se empleó la técnica de reflectancia total atenuada (ATR); y 4) Patrón de difracción de rayos X (DRX) tomado en un difractometro X-Pert Pro marca PANalytical.

\section{Cálculo de la energía de banda prohibida óptica $\left(E_{g}^{o p}\right)$ y el diámetro de partícula}

Para el cálculo de la energía de la banda prohibida óptica se utilizó el método gráfico de Tauc, el cual arroja una mejor aproximación que la extrapolación directa del espectro UV-vis para hallar el $\lambda$ de inicio de la absorción (Mi et al, 2012). La Figura 1 es un ejemplo del método gráfico de Tauc usado para calcular la $\mathrm{E}_{\mathrm{g}}^{\mathrm{op}}$ de los PCs de CdSe/MSA crecidos hasta $3 \mathrm{~h}$.

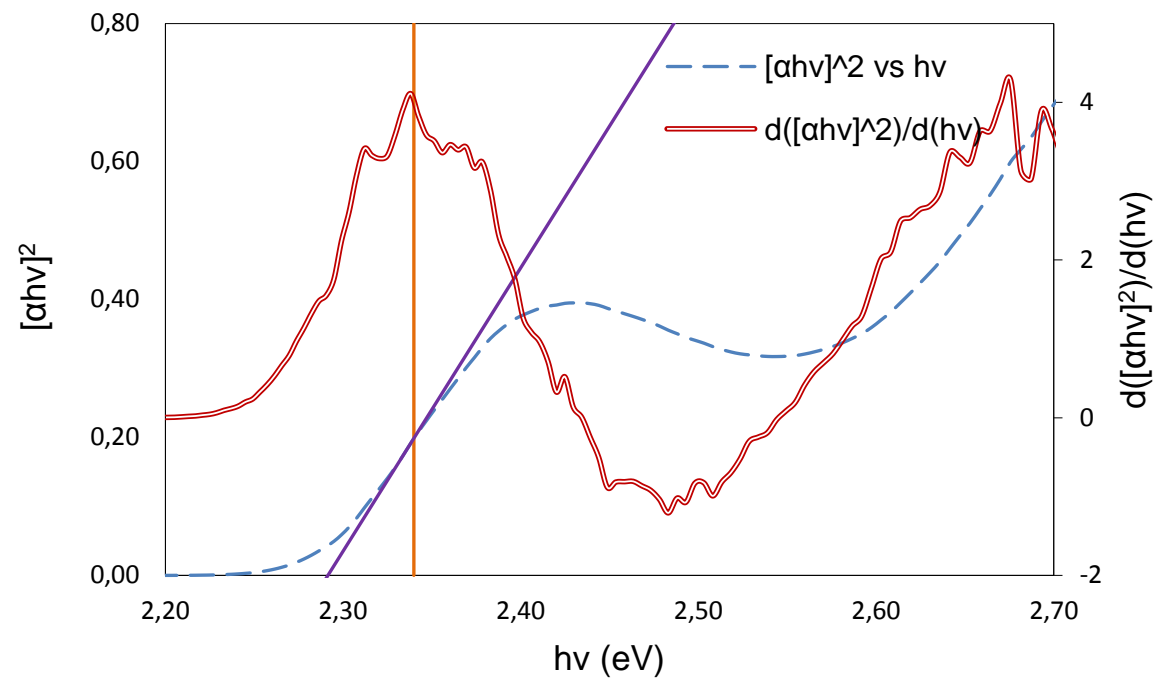

Fig. 1: Ejemplo del método gráfico de Tauc para calcular la energía de banda prohibida óptica $\left(E_{g}\right)$

La aplicación de dicho método parte de la siguiente expresión del fenómeno de absorción:

$$
\alpha(h v)=C\left(h v-E_{g}^{o p}\right)^{1 / 2}, \quad\left(h v \geq E_{g}^{o p}\right)
$$

Donde $\alpha$ es el coeficiente de absorción, definido como el cociente de la absorbancia y la longitud de trayectoria del rayo de luz (A/L), C es una constante, hv es la energía de los fotones incidentes, el valor del exponente $\mathrm{r}=1 / 2$ denota la naturaleza de la transición electrónica, y $\mathrm{E}_{\mathrm{g}}^{\mathrm{op}}$ es la energía de banda prohibida óptica de las nanopartículas.

Para el cálculo del diámetro medio de los PCs (núcleo nanocristalino) se empleó la ecuación 2 de Yu et al., (2003).

$$
\mathrm{D}=\left(1.6122 \times 10^{-9}\right) \lambda^{4}-\left(2.6575 \times 10^{-6}\right) \lambda^{3}+\left(1.6242 \times 10^{-3}\right) \lambda^{2}-(0.4277) \lambda+41.57
$$

La ecuación anterior es una función polinómica de ajuste de diámetros medidos con TEM y DRX, donde el diámetro $\mathrm{D}(\mathrm{nm})$ es función de la longitud de onda $\lambda(\mathrm{nm})$ del 1er pico de absorción excitónico. Los intervalos de aplicación de la ecuación son $\lambda(320-660) \mathrm{nm}$ y $\mathrm{D}(0.75-8.5) \mathrm{nm}$. 


\section{Evaluación fotocatalítica de los PCs}

Para evaluar la evolución de $\mathrm{H}_{2}$ por actividad fotocatalítica se sintetizaron 5 tipos de PCs en polvo: 4 tamaños diferentes de PCs de CdSe/MSA y 1 tamaño de PCs de CdSe/TGA, cuyas propiedades se describen en la Tabla 1. Sólo se escogieron PCs de CdSe/TGA crecidos hasta $8 \mathrm{~h}$ ya que estos mostraron picos de absorción y emisión más claros, así como el menor ATMM.

Tabla 1: Propiedades de los PCs preparados para ensayos en fotolisis de $\mathrm{H}_{2} \mathrm{O}$

\begin{tabular}{cccccc}
\hline $\begin{array}{c}\text { Tipo de } \\
\text { envolvente }\end{array}$ & $\begin{array}{c}\text { 1er pico de abs } \\
\text { excitónico }(\mathrm{nm})\end{array}$ & $\begin{array}{c}\text { Diámetro }(\mathrm{nm}) \\
{[\text { Peng] }}\end{array}$ & $\begin{array}{c}\mathrm{E}_{\mathrm{g}}^{\mathrm{op}}(\mathrm{eV}) \\
{[\text { Tauc }]}\end{array}$ & $\begin{array}{c}\text { Pico de emisión } \\
(\mathrm{nm})\end{array}$ & $\begin{array}{c}\text { ATMM } \\
(\mathrm{nm})\end{array}$ \\
\hline TGA & 484 & 2.21 & 2.38 & 608 & 80.0 \\
MSA & 471 & 2.10 & 2.48 & 495 & 38.6 \\
MSA & 500 & 2.35 & 2.36 & 519 & 37.8 \\
MSA & 517 & 2.53 & 2.29 & 536 & 34.0 \\
MSA & 525 & 2.63 & 2.25 & 546 & 39.2 \\
\hline
\end{tabular}

Los PCs seleccionados tuvieron una $\mathrm{E}_{\mathrm{g}}^{\mathrm{op}}$ en el intervalo 2.25-2.48 eV. Según la teoría de Gerischer (1990) a mayor energía de banda prohibida se consiguen mayores tasas de transferencia de carga, como ha sido confirmado posteriormente (Zhao et al, 2013). Sin embargo, existe un límite $\mathrm{E}_{\mathrm{g}-\mathrm{lim}} \approx 2.48 \mathrm{eV}$, tal que si $\mathrm{E}_{\mathrm{g}}>$ $\mathrm{E}_{\mathrm{g}-\mathrm{lim}}$ la tasa de evolución de $\mathrm{H}_{2}$ empieza a disminuir. Tal afirmación se debe a que la intensidad del espectro solar en la superficie terrestre disminuye para longitudes de onda (UV) menores a $500 \mathrm{~nm}$ (pico máximo).

Las semirreacciones y la reacción global que tendrían lugar entre el catalizador, el donante de sacrificio y el $\mathrm{H}_{2} \mathrm{O}$ se presentan a continuación.

$$
\begin{array}{lr}
\mathrm{H}_{2} \mathrm{O}+\mathrm{SO}_{3}^{2-} \rightarrow 2 \mathrm{H}^{+}+\mathrm{SO}_{4}^{2-}+2 \mathrm{e}^{-} & \mathrm{E}(\mathrm{pH} 9.6)=-1.05 \mathrm{~V} \text { vs. NHE } \\
2 \mathrm{H}^{+}+2 \mathrm{e}^{-} \rightarrow \mathrm{H}_{2} & \mathrm{E}(\mathrm{pH} 9.6)=-0.43 \mathrm{~V} \text { vs. NHE } \\
\mathrm{H}_{2} \mathrm{O}+\mathrm{SO}_{3}^{2-}--(\mathrm{hv} / \mathrm{CdSe} \mathrm{PC})-\rightarrow \mathrm{H}_{2}+\mathrm{SO}_{4}^{2-}
\end{array}
$$

Fue necesario recalcular los potenciales electroquímicos usando la ecuación de Nernst con la condición de $\mathrm{pH}=9.6$, medido en las soluciones de PCs de $\mathrm{MSA} / \mathrm{Na}_{2} \mathrm{SO}_{3}$ (condiciones iniciales). En este caso, el azufre del sulfito dona un par de electrones y cambia su estado de oxidación de $+4 a+6$. La reacción de formación de $\mathrm{O}_{2}$ es sustituida por la oxidación del $\mathrm{SO}_{3}^{2-}$ (donador electrónico), para evitar la elevada sobretensión de la oxidación del $\mathrm{H}_{2} \mathrm{O}: \mathrm{E}^{\circ}\left[\mathrm{O}_{2} / \mathrm{H}_{2} \mathrm{O}\right]=1.23 \mathrm{~V}$.

\section{Montaje experimental de la fotólisis del $\mathrm{H}_{2} \mathrm{O}$}

El montaje utilizado se muestra en la Figura 2, el cual consiste en un fotorreactor con vaso de inmersión hecho en borosilicato, una plancha de agitación, un termostato de refrigeración (Lauda RA12), cilindro de $\mathrm{N}_{2}$ conectado al sistema, detector de $\mathrm{H}_{2}$ marca Crowcon (Tetra / 0-2000 ppm), lámpara de arco-xenón de $55 \mathrm{~W}$ (HID $9005-6000 \mathrm{~K}$ ) y fuente de poder (CA a CC).

Puesto que la lámpara se introduce dentro del vaso de inmersión, la función de las paredes de borosilicato es reflejar luz que provoque la descomposición directa del sulfito $(<320 \mathrm{~nm})$. Adicionalmente, el vaso de inmersión posee una camisa refrigerante donde fluye agua bombeada desde el termostato. El aprovechamiento de la energía solar sugiere el uso de fuentes de radiación que simulen bien el espectro solar, por lo cual se escogió la lámpara de xenón cuyo espectro es más aproximado que el de lámparas halógenas o de mercurio. Empleando un radiómetro SM206 se midió la radiación incidente en la solución en un promedio de $150 \mathrm{~mW} / \mathrm{cm}^{2}$. 


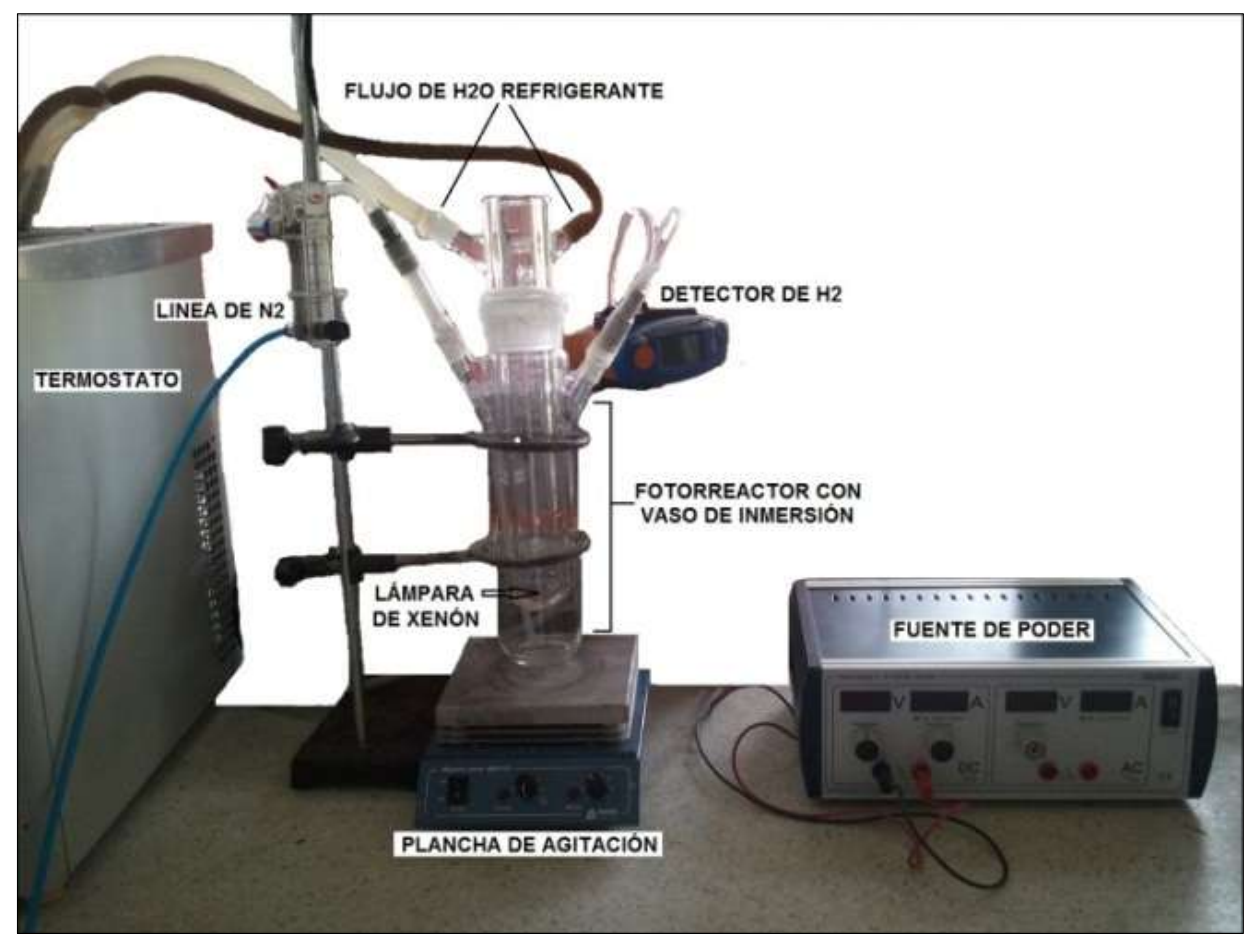

Fig. 2: Montaje experimental para medir actividad fotocatalítica de los PCs

$50 \mathrm{mg}$ de cada tipo de PCs fue dispersado en $100 \mathrm{~mL}$ de solución $0.1 \mathrm{M}$ de $\mathrm{Na}_{2} \mathrm{SO}_{3}$ (preparada con agua desionizada). Una vez vertida la solución acuosa de los PCs dentro del fotorreactor, se realizó burbujeo de $\mathrm{N}_{2}$ durante 30 min para extraer la mayor cantidad posible de $\mathrm{O}_{2}$ en dilución y de la cabeza del reactor. Posteriormente se empezó a irradiar con la lámpara de xenón y se inició el conteo de tiempo. El detector tetra conectado en línea al sistema permitió la medición directa de la concentración de $\mathrm{H}_{2}$ en la cabeza de gas del fotorreactor. Dado que la capacidad de la bomba interna del detector es muy baja, la succión y la descarga deben igualarse en presión conectando ambas al sistema. De esta forma, la lectura de $\mathrm{H}_{2}$ en ppm fue registrada cada hora durante un periodo de radiación total de 5 a 6 horas. Finalmente, para el cálculo de las moles de $\mathrm{H}_{2}$ se empleó la ecuación de estado de los gases y la ley de Amagat para una mezcla, de donde se obtiene la expresión de la ecuación 6.

$$
\mathrm{N}_{\mathrm{H} 2}=\mathrm{x}_{\mathrm{H} 2} \frac{\mathrm{PV}}{\mathrm{RT}}
$$

En la derivación de la expresión se consideró gas ideal dado que el factor de compresibilidad del $\mathrm{H}_{2}$ es aproximadamente igual a 1 alrededor de condiciones atmosféricas (Perry 1997). $\mathrm{x}_{\mathrm{H} 2}$ es la fracción volumétrica en $\mathrm{m}^{3} / \mathrm{m}^{3}$ calculada a partir de la concentración medida en $\mathrm{ppm}\left(\mathrm{cm}^{3} / \mathrm{m}^{3}\right)$. Posteriormente los datos experimentales se graficaron en curvas de: $\mu \mathrm{mol} \mathrm{H}_{2}$ vs. tiempo, y se calcularon las tasas de evolución correspondientes al tramo lineal de las curvas.

\section{RESULTADOS Y DISCUSIÓN}

\section{Reacción del precursor del Se-2}

Durante la reacción $\mathrm{NaBH}_{4}+\mathrm{Na}_{2} \mathrm{SeO}_{3}$ el aumento del pH es una prueba de la formación del ión seleniuro Se${ }^{2}$ puesto que este prevalece únicamente en condiciones muy básicas. En condiciones neutras es más común encontrar iones de seleniuro de hidrógeno $\mathrm{HSe}^{-}$, y en condiciones ácidas se forma el seleniuro de hidrógeno $\mathrm{H}_{2} \mathrm{Se}$.

Según el comportamiento del pH de la solución existe un tiempo donde la concentración de $\mathrm{Se}^{-2}$ alcanzaría un valor máximo. Para una relación molar $\mathrm{NaBH}_{4} / \mathrm{Na}_{2} \mathrm{SeO}_{3}: 1.32 / 0.15$ y un volumen de reacción de $5 \mathrm{~mL}$ dicho tiempo fue de 6.2 min de reacción, el cual se interpoló de la gráfica pH vs. tiempo de la Figura 3 . En otros estudios el punto de referencia para la mezcla de los precursores ha sido cualitativo, esto es, cuando la solución de $\mathrm{Se}^{-2}$ alcanza un color rojo parduzco. 


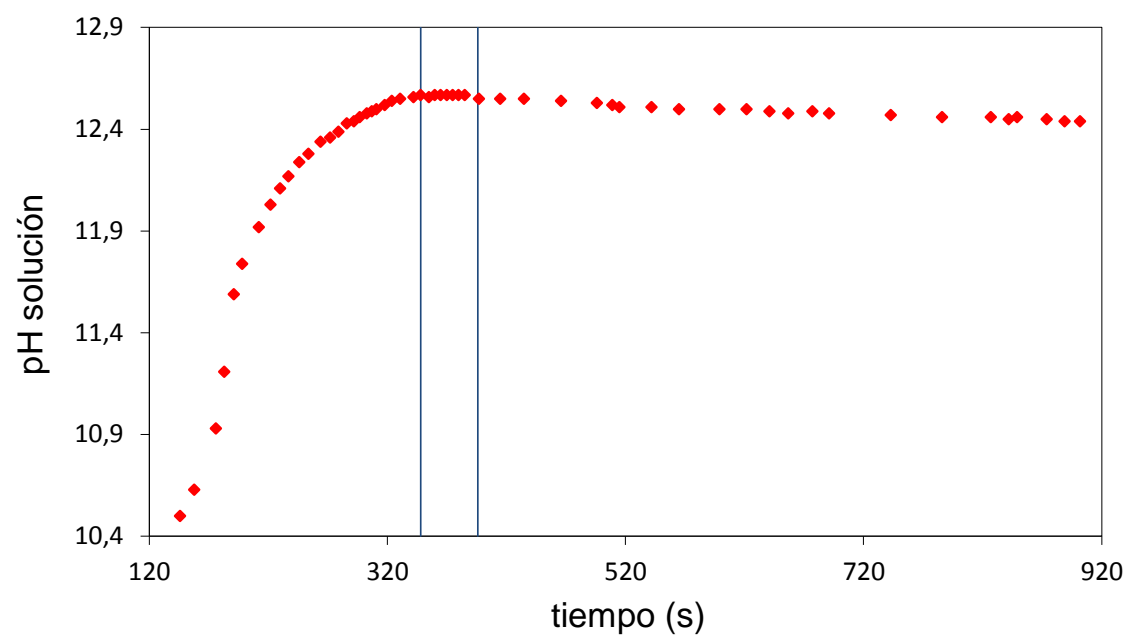

Fig. 3: pH vs. tiempo de la reacción de formación del Se-2

\section{Espectros de absorción UV-vis y de fluorescencia}

Una vez terminado el periodo de reflujo, se midió el espectro UV-vis y el espectro PL de cada muestra colectada. En ambos casos (MSA y TGA), los picos de absorción de los NCs sintetizados mostraron confinamiento cuántico al presentar cambios hacia el azul en el espectro visible, considerando que la energía de banda prohibida del CdSe en bloque es de $1.73 \mathrm{eV}\left(\lambda_{\text {abs }} \sim 718 \mathrm{~nm}\right)$. Como se observa en la Figura 4, los PCs de CdSe/MSA muestran picos de absorción y emisión bien definidos. Mientras que el 1er pico de absorción excitónico cambió durante el periodo de reflujo desde $492 \mathrm{~nm}$ hasta $525 \mathrm{~nm}$, el pico de emisión cambió en el intervalo de 514 a $546 \mathrm{~nm}$ (Tabla 2). El cálculo del ATMM de los espectros PL arrojó en promedio un valor de $39.7 \mathrm{~nm}(1-8 \mathrm{~h})$, el cual indica una distribución de tamaños bastante estrecha comparada con la distribución correspondiente a la solución cruda (113 nm).

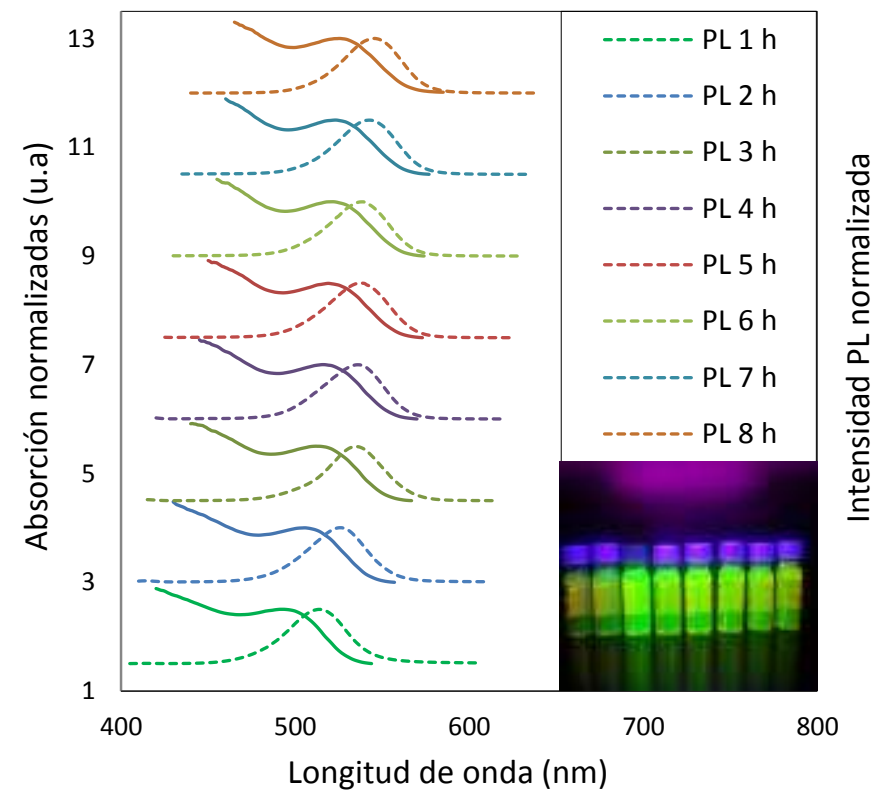

Fig. 4: Espectros PL (---) y de absorción UV-vis (-) de PCs de CdSe/MSA entre 1- $8 \mathrm{~h}$ de reflujo

El cambio de Stokes equivalente a la diferencia de longitudes de onda entre el pico de emisión y el pico de absorción fue en promedio $20.4 \mathrm{~nm}$, lo cual sugiere que la fluorescencia de los PCs de CdSe/MSA es atribuible a la banda prohibida o emisión excitónica. 
Tabla 2: Picos de absorción y de emisión de los PCs de CdSe/MSA. ATMM de los espectros PL

\begin{tabular}{cccc}
\hline $\begin{array}{c}\text { Tiempo de } \\
\text { reflujo } \\
(\mathrm{h})\end{array}$ & $\begin{array}{c}\text { 1er pico de } \\
\text { absorción excitónico } \\
(\mathrm{nm})\end{array}$ & $\begin{array}{c}\text { Pico de } \\
\text { emisión } \\
(\mathrm{nm})\end{array}$ & $\begin{array}{c}\text { ATMM } \\
(\mathrm{nm})\end{array}$ \\
\hline & & & \\
1 & 492 & 514 & 40.0 \\
2 & 505 & 526 & 39.7 \\
3 & 512 & 535 & 37.5 \\
4 & 516 & 536 & 41.2 \\
5 & 519 & 537 & 41.2 \\
6 & 521 & 539 & 39.4 \\
7 & 523 & 543 & 39.8 \\
8 & 525 & 546 & 39.2 \\
& & & \\
\hline \multicolumn{4}{c}{ Promedio aritmético $(1-8 \mathrm{~h})$} \\
\hline
\end{tabular}

Los espectros y picos característicos de los PCs de CdSe/TGA después de 1 y $8 \mathrm{~h}$ de reflujo se muestran en la Figura 5 y la Tabla 3 respectivamente. En este caso el primer pico de absorción no estaba bien definido como en el caso de los PCs/MSA. Adicionalmente, la distribución de tamaños asociada al espectro PL fue demasiado amplia aún después del reflujo de $8 \mathrm{~h}$ (ATMM igual a $80 \mathrm{~nm}$ ).

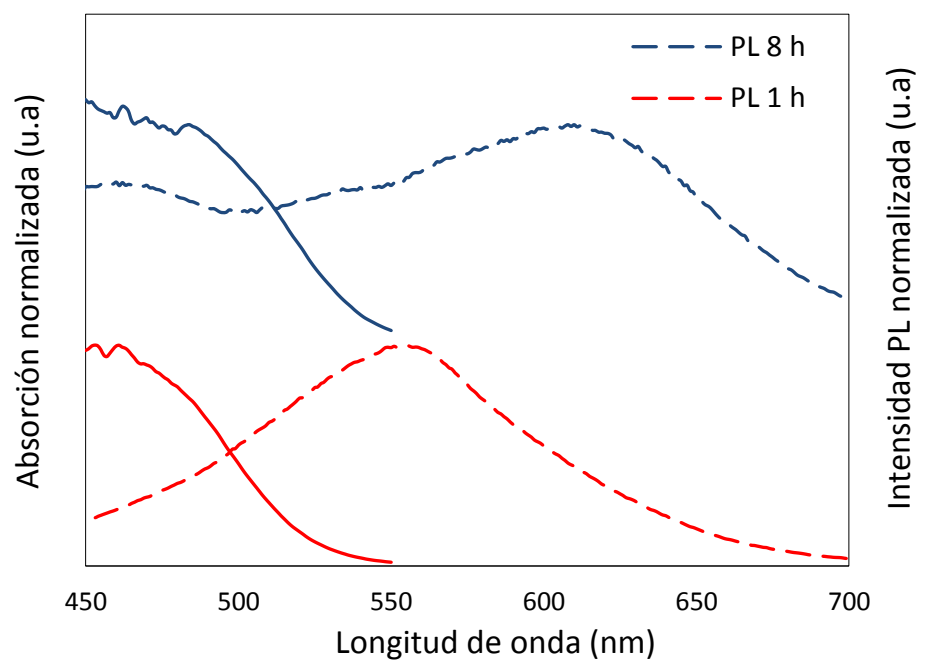

Fig. 5: Espectros PL (---) y de absorción UV-vis (-) de PCs de CdSe/TGA en 1 h y 8 h de reflujo

Tabla 3: Picos de absorción y de emisión de los PCs de CdSe/TGA. ATMM de los espectros PL

\begin{tabular}{cccc}
\hline $\begin{array}{c}\text { Tiempo de } \\
\text { reflujo } \\
(\mathrm{h})\end{array}$ & $\begin{array}{c}\text { 1er pico de } \\
\text { absorción excitónico } \\
(\mathrm{nm})\end{array}$ & $\begin{array}{c}\text { Pico de } \\
\text { emisión } \\
(\mathrm{nm})\end{array}$ & $\begin{array}{c}\text { ATMM } \\
(\mathrm{nm})\end{array}$ \\
\hline 1 & 461 & 555 & 94.7 \\
8 & 484 & 608 & 80.0 \\
\hline
\end{tabular}

De acuerdo con Morris y Zubkov (2014) en el caso de cadenas lineales cortas como el TGA, durante la síntesis el transporte de monómeros nuevos a través de la envolvente es más fácil y rápido, lo cual produce PCs con amplio ATMM y un alto índice de defectos en la superficie cristalina.

Ambos tipos de soluciones de PCs fueron irradiadas bajo una lámpara con UV de $365 \mathrm{~nm}$. Los PCs de CdSe/MSA emitieron fluorescencia en el verde, la cual aumentó desde la solución de $1 \mathrm{~h}$ hasta la solución de $3 \mathrm{~h}$ de reflujo y luego decayó como se observa en la foto insertada en la Figura 4. Esta observación es 
consistente con un rendimiento cuántico de fotoluminiscencia (PL-QY) máximo del 18\%, medido por Wang et al., (2012) para PCs de $3 \mathrm{~h}$ de reflujo. Por otro lado la fluorescencia de los PCs de CdSe/TGA fue casi imperceptible y de color tenue amarillo, el cual corresponde bien al pico de emisión de $608 \mathrm{~nm}$ y a otras longitudes de onda dada la amplia su distribución de tamaños (Figura 5).

\section{Espectro infrarrojo por transformada de Fourier (FTIR)}

Para analizar la composición superficial de los NCs con distinta capa envolvente se midió el espectro FTIR de PCs de CdSe/TGA crecidos hasta 8 h (Fig. 6a), y de PCs de CdSe/MSA crecidos durante 8 h (Fig. 6b).
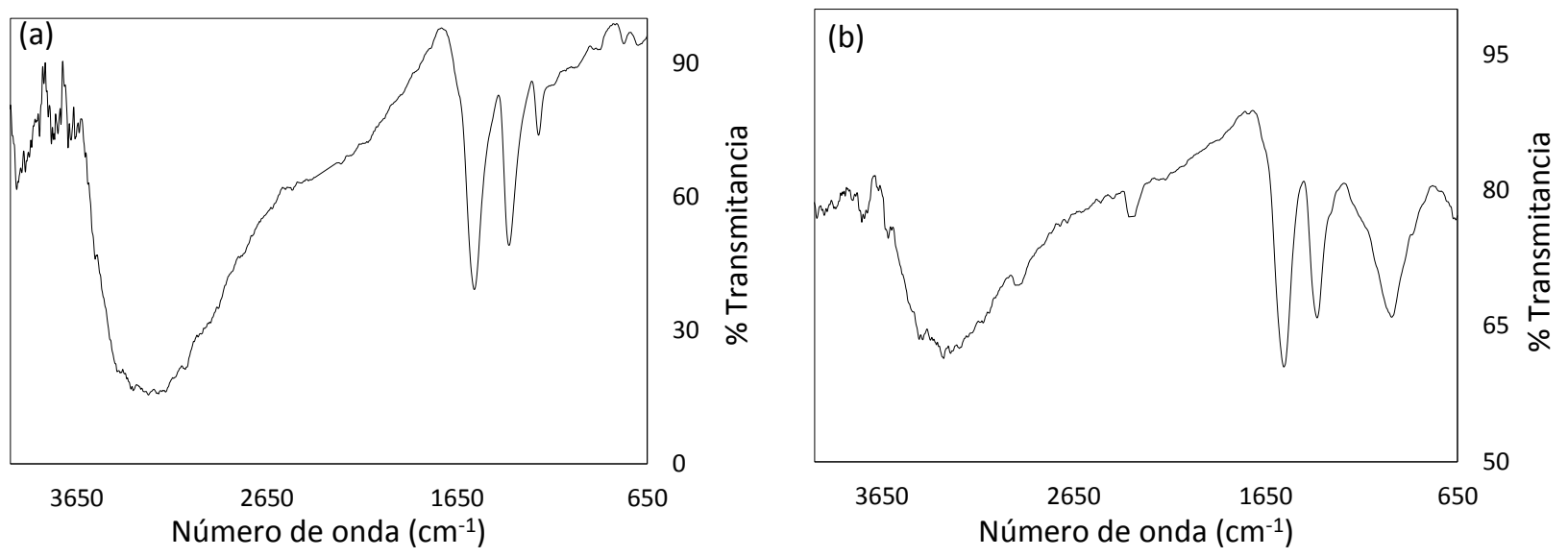

Fig. 6: (a) Espectro FTIR de polvo de PCs de CdSe/TGA crecidos durante $8 \mathrm{~h}$ a $98^{\circ} \mathrm{C}$. (b) Espectro FTIR de polvo de PCs de CdSe/MSA crecidos durante $8 \mathrm{~h}$ a $98^{\circ} \mathrm{C}$

En ambos casos es notoria la banda de absorción asignada al $\mathrm{OH}$ del agua adsorbida por las muestras: $3274.54 \mathrm{~cm}^{-1}$ para PCs con TGA y $3331.43 \mathrm{~cm}^{-1}$ para PCs con MSA. La banda de absorción característica de la vibración $\mathrm{C}=\mathrm{O}$ en ácidos $\left(1715 \mathrm{~cm}^{-1}\right)$ parece haber sido desplazada por la presencia de iones carboxilato (R-COO-) en la superficie de ambos tipos de PCs, los cuales registran picos de absorción en: $1560.13 \mathrm{~cm}^{-1}$ para PCs/TGA y $1553.38 \mathrm{~cm}^{-1}$ para PCs/MSA. Al precipitar los nanocristales es posible que no toda la carga superficial sea neutralizada debido a que los iones carboxilato presentan estructuras de resonancia relativamente estables.

Por último, en ninguno de los espectros se observa la vibración del grupo S-H que corresponde a una banda de absorción observada en $2560 \mathrm{~cm}^{-1}$. Estos resultados sugieren que los 2 agentes estabilizantes coordinaron con átomos de $\mathrm{Cd}$ presentes en la superficie del núcleo nanocristalino a través del enlace Cd-S y, del otro extremo, los grupos carboxílicos confieren una carga negativa a los PCs en solución al disociarse en iones carboxilato.

\section{Difracción de rayos $X(D R X)$}

Esta técnica se aplicó en una muestra en polvo de PCs de CdSe/MSA crecidos durante $8 \mathrm{~h}$ a $98^{\circ} \mathrm{C}$. Al observar el patrón DRX de la Figura 7 se identificaron 3 características de la estructura cubica blenda de zinc (ZnS), es decir, 3 picos en la posición 20: 26, 43.4 y 51.2 que corresponden aproximadamente a las reflexiones: (111), (220) y (311). La estructura cubica es de esperarse para PCs sintetizados a bajas temperaturas, mientras que la estructura wurzita es observada en PCs sintetizados a altas temperaturas (> $200^{\circ} \mathrm{C}$ ) (Wang et al, 2012). Los picos que presenta el patrón DRX son anchos comparados a los de materiales en bloque debido al tamaño finito de los nanocristales.

\section{Curvas de crecimiento de los PCs}

Remplazando la longitud de onda del 1er pico de absorción excitónico en la ecuación 2 de Yu et al., (2003), se calcularon los diámetros de las muestras tomadas durante el reflujo en intervalos de 1 hora. Al graficar el diámetro de los PCs de CdSe/MSA en función del tiempo se obtuvo una curva creciente como se observa en la Figura 8a. Dado que en confinamiento cuántico cada tamaño de PC corresponde a una banda prohibida específica, también se construyó la curva de la energía de banda óptica vs. tiempo para los PCs de $\mathrm{CdSe} / \mathrm{MSA}$. Como es de esperarse $\mathrm{E}_{\mathrm{g}}^{\mathrm{op}}$ en función del tiempo es una curva decreciente (Figura 8b), debido a que el diámetro aumenta y $\mathrm{E}_{\mathrm{g}}^{\mathrm{op}}$ disminuye en el régimen de confinamiento cuántico. La curva $\mathrm{E}_{\mathrm{g}}^{\mathrm{op}}$ vs. $\mathrm{t}$ es útil 
cuando se requiere sintetizar PCs con una $\mathrm{E}_{\mathrm{g}}^{\mathrm{op}}$ predeterminada para el acoplamiento de bandas electrónicas en varios tipos de celda.

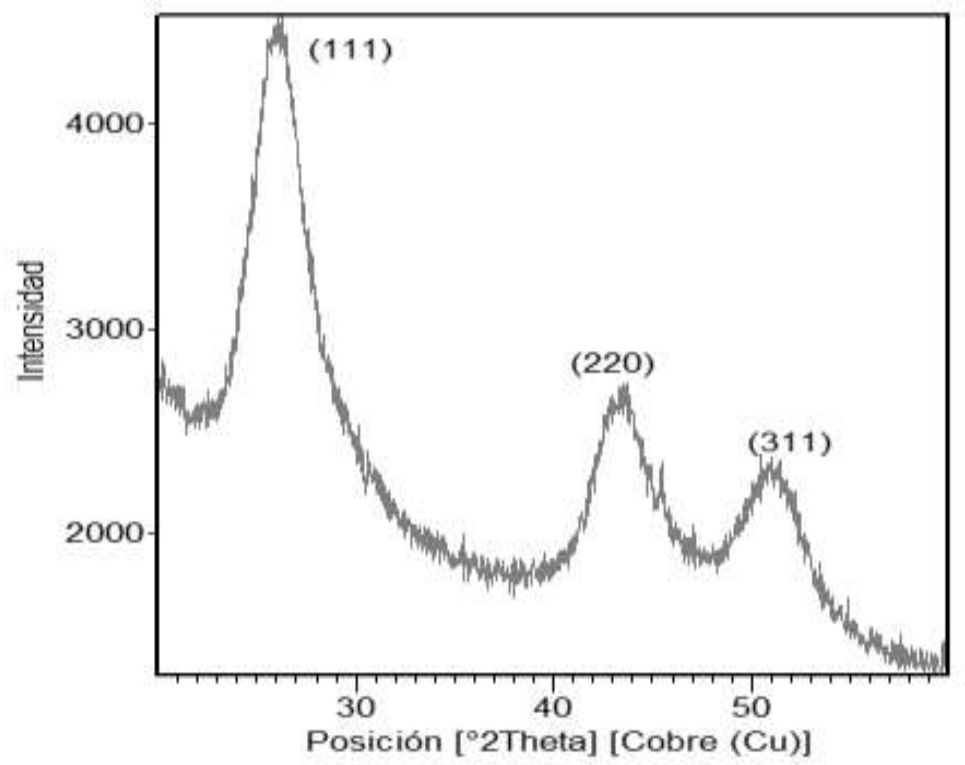

Fig. 7: DRX de polvo de PCs de CdSe/MSA crecidos a $98^{\circ} \mathrm{C}$ hasta 8 de reflujo
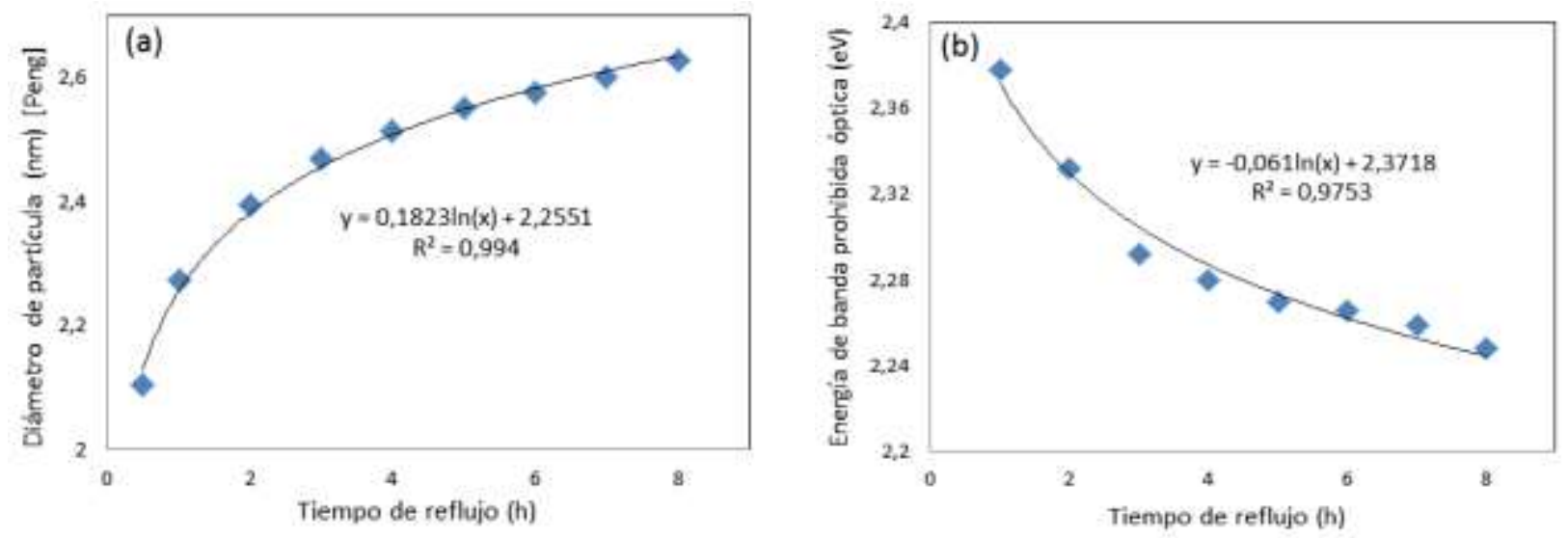

Fig. 8: (a) Curva de crecimiento de PCs de CdSe/MSA a $98^{\circ} \mathrm{C}$ (b) Energía de banda prohibida óptica vs. tiempo de reflujo de PCs de CdSe/MSA

La formación de PCs coloidales se puede dividir en tres etapas: nucleación, crecimiento y maduración. Durante la nucleación, la concentración de monómero líquido (reactivos o precursores) es parcialmente agotada por la formación de muchos núcleos de aproximadamente el mismo tamaño. Luego, las partículas crecen por el consumo del monómero. Por último, como el monómero se agota, el tamaño promedio aumenta por crecimiento competitivo o maduración de Ostwald, en el cual las partículas más grandes crecen mientras que las partículas más pequeñas se encogen y desaparecen, para reducir la energía superficial neta del sistema (Dickerson et al, 2005). Durante el reflujo, los PCs de CdSe/MSA alcanzaron el mínimo ATMM (37 $\mathrm{nm}$ ) y el máximo PL-QY simultáneamente en un tiempo de reflujo de $3 \mathrm{~h}$, condición en la cual las nanopartículas están prácticamente en equilibrio con la concentración de monómeros del seno (Qu y Peng. 2002). También se notó un aumento repentino del ATMM (Tabla 2) y una disminución de la tasa de crecimiento (Figura 8a) después de $3 \mathrm{~h}$ de reflujo, lo cual es característico de la etapa de maduración de Ostwald debido a la escasez de reactivos precursores.

Como se indicó anteriormente el 1er pico de absorción excitónico de los PCs de CdSe/TGA no está claramente definido en los espectros de absorción, por tanto solo se presentan los valores promedio del diámetro de partícula y la energía de banda prohibida al inicio y al final del periodo de reflujo de 8 horas (Tabla 4). 
Tabla 4: Diámetro medio y energía de banda prohibida óptica de los PCs de CdSe/TGA

\begin{tabular}{cccc}
\hline & $\begin{array}{c}\text { 1er pico de abs } \\
\text { excitónico }(\mathrm{nm})\end{array}$ & $\begin{array}{c}\text { Diámetro }(\mathrm{nm}) \\
\text { [Peng] }\end{array}$ & $\begin{array}{c}\mathrm{E}_{\mathrm{g}}^{\mathrm{op}}(\mathrm{eV}) \\
{[\mathrm{Tauc}]}\end{array}$ \\
\hline 1 & 461 & 2.03 & 2.53 \\
8 & 484 & 2.21 & 2.38 \\
\hline
\end{tabular}

\section{Evolución fotocatalítica de $\mathrm{H}_{2}$}

En la Figura 9 se compara la evolución de $\mathrm{H}_{2}$ de los 4 tamaños escogidos de PCs de CdSe/MSA, de los cuales, los PCs de $2.53 \mathrm{~nm}$ y $2.63 \mathrm{~nm}$ tuvieron la tasa de evolución de $\mathrm{H}_{2}$ más baja y los PCs de $2.1 \mathrm{~nm}$ presentaron una tasa máxima de $1.126 \mu \mathrm{mol} \mathrm{H}_{2} / \mathrm{h}$.

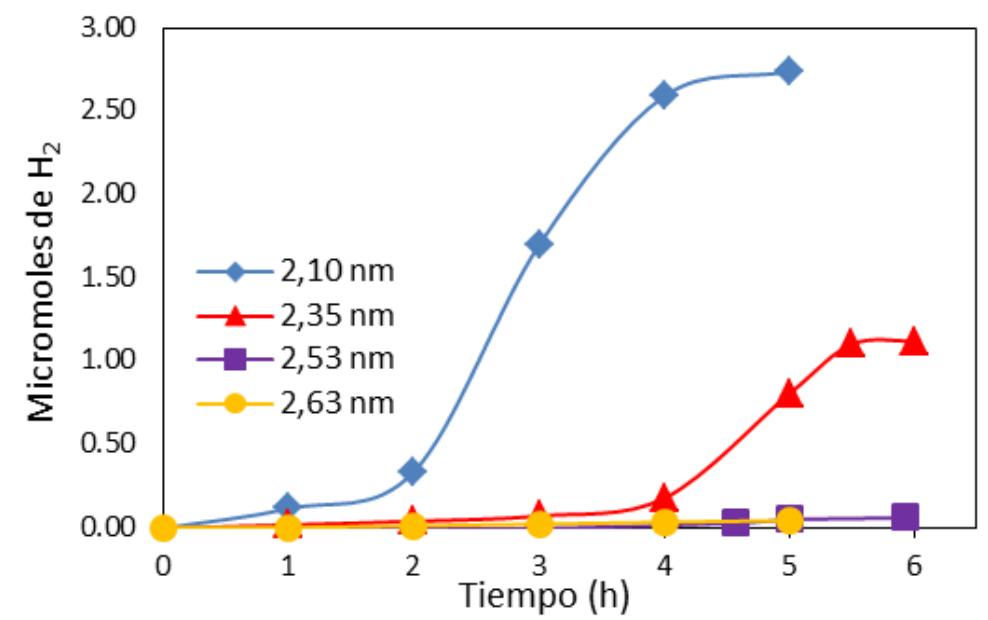

Fig. 9: Evolución de $\mathrm{H}_{2}$ en 4 soluciones con diferentes tamaños de PCs de CdSe/MSA

Tabla 5: Tasas de evolución de $\mathrm{H}_{2}$ en 4 soluciones con diferentes tamaños de PCs de CdSe/MSA

\begin{tabular}{cccc}
\hline $\begin{array}{c}\text { Diámetro } \\
\text { medio de } \\
\text { partícula } \\
(\mathrm{nm})\end{array}$ & $\begin{array}{c}\mathrm{E}_{\mathrm{g}}^{\text {op }} \\
(\mathrm{eV})\end{array}$ & $\begin{array}{c}\text { Tasa de } \\
\text { evolución } \\
(\mu \mathrm{mol} \mathrm{H} / \mathrm{h})\end{array}$ & $\begin{array}{c}\text { Periodo } \\
\text { tasa } \\
\text { constante } \\
(\mathrm{h})\end{array}$ \\
\hline 2.10 & 2.48 & 1.126 & $2-4$ \\
2.35 & 2.36 & 0.618 & $4-5.5$ \\
2.53 & 2.29 & 0.023 & $3-6$ \\
2.63 & 2.25 & 0.011 & $1-5$ \\
\hline
\end{tabular}

En todos los casos la solución de PCs se mantuvo a $28^{\circ} \mathrm{C}$, temperatura de equilibrio alcanzada por efecto de la refrigeración con agua $\left(25^{\circ} \mathrm{C}\right)$ y el calentamiento por radiación infrarroja de la lámpara de xenón. Para todos los tamaños se observó un periodo inicial entre 1 y $4 \mathrm{~h}$ donde la tasa de evolución fue muy baja. Posteriormente, la pendiente aumentó y se mantuvo constante en un periodo de 2 a $4 \mathrm{~h}$, las tasas calculadas corresponden a este periodo (Tabla 5).

La tasa de evolución obtenida por Holmes et al., (2011) para PCs de CdSe/MCE con 2.2 nm fue $6.096 \mu$ mol $\mathrm{H}_{2} / \mathrm{h}$. Aunque se empleó la misma cantidad de catalizador, igual concentración de $\mathrm{Na}_{2} \mathrm{SO}_{3}$ y el mismo volumen de solución, esta tasa no es directamente comparable con la tasa obtenida para PCs de CdSe/MSA (2.1 nm) del presente estudio, puesto que la fuente de irradiación usada por Holmes et al., (2011) tenía una potencia de $300 \mathrm{~W}$ (5.5 veces mayor) lo cual debe aumentar sustancialmente la tasa de fotogeneración de portadores de carga. No obstante, un aspecto más importante es que en PCs de CdSe/MCE se observó evolución de $\mathrm{H}_{2}$ hasta por 24 horas, mientras que en los PCs de CdSe/MSA la evolución de $\mathrm{H}_{2}$ se vio interrumpida después de un periodo en torno a $5 \mathrm{~h}$. 
Tanto en los PCs de $2.1 \mathrm{~nm}$ como los de $2.35 \mathrm{~nm}$ se observó una interrupción de la evolución de $\mathrm{H}_{2}$ después de 5 y 5.5 h respectivamente, y en ambos casos la solución cambió lentamente de color naranja a café. Según Zhao et al., (2013), este resultado corresponde con la reducción de $\mathrm{Cd}^{+2}$ y la formación abundante de $\mathrm{Cd}^{(0)}$ en la red cristalina y la superficie de los PCs durante cierto periodo inicial ( 0.5 a 3 h). También se demostró que el grado de formación de $\mathrm{Cd}^{(0)}$ dependía de la banda electrónica de los PCs, el cual aumentó en la medida que la diferencia entre el potencial de reducción del $\mathrm{Cd}^{+2}$ y la banda de conducción mínima (BCM) de los PCs se hizo más negativa. Según este resultado, la presencia de cadmio metálico favorece la conducción desde el interior del semiconductor y la captura de electrones en sitios superficiales aumentando la tasa de reducción de protones $\mathrm{H}^{+}$. En el caso presente, de acuerdo al análisis realizado el periodo de improductividad inicial de las curvas de evolución no se debe sólo a la formación de $\mathrm{Cd}^{(0)}$, sino también a la difusión limitada del $\mathrm{SO}_{3}^{2-}$ a través de la capa envolvente de los PCs/MSA.

La interrupción temprana de la evolución de $\mathrm{H}_{2}$ se atribuyó a la naturaleza de la envolvente de MSA y a un PL-QY relativamente alto. El grupo - $\mathrm{SH}$ del $\mathrm{MSA}\left[\mathrm{HO}_{2} \mathrm{CCH}_{2} \mathrm{CH}(\mathrm{SH}) \mathrm{CO}_{2} \mathrm{H}\right]$ esta enlazado a un carbono intermedio de la cadena, a diferencia de otros agentes como el MCE y el TGA en los cuales el grupo -SH esta enlazado a un extremo de la cadena. Esta característica hace que el MSA tenga dos ramificaciones una vez enlazado a la superficie de los PCs por medio del enlace Cd-S. Por tanto, el MSA tiene un grupo carboxilato (-COO-) en cada ramificación, lo cual puede crear un mayor impedimento estérico entre los ligantes unidos a la superficie, y producir cierta repulsión sobre iones negativos que intenten llegar a (o salir desde) la superficie del cristal.

De acuerdo con lo anterior, si se asume agitación lenta y se desprecia la migración de especies iónicas, la evolución de $\mathrm{H}_{2}$ en PCs de CdSe/MSA con PL-QY $\geq 4 \%$ es limitada por la difusión. Por lo tanto, en el periodo de la tasa de evolución constante la velocidad de reacción se puede tomar igual a la velocidad de transferencia de masa $\left(\mathrm{v}_{\mathrm{rxn}}=\mathrm{v}_{\mathrm{tm}}\right)$, y el sistema se simplifica al modelo de la capa estancada o capa de difusión de Nernst. La reacción de fotocorrosión de los huecos compite con la reducción de los huecos por parte del $\mathrm{SO}_{3}^{2-}$, por lo cual, si la velocidad de reducción de los huecos es limitada por la transferencia de masa del donor $\mathrm{SO}_{3}^{2-}$, la velocidad de fotocorrosión de los PCs se hace mayor. La fotocorrosión superficial produce la eliminación de la estructura envolvente, lo que origina la aglomeración y precipitación de los PCs y por ende, la interrupción de la evolución de $\mathrm{H}_{2}$.

\section{Aumento de la temperatura y cambio de la capa envolvente}

Se llevó a cabo una prueba de los PCs con $2.1 \mathrm{~nm}$ a $50^{\circ} \mathrm{C}$ y su curva de evolución se comparó con la obtenida a $28^{\circ} \mathrm{C}$.
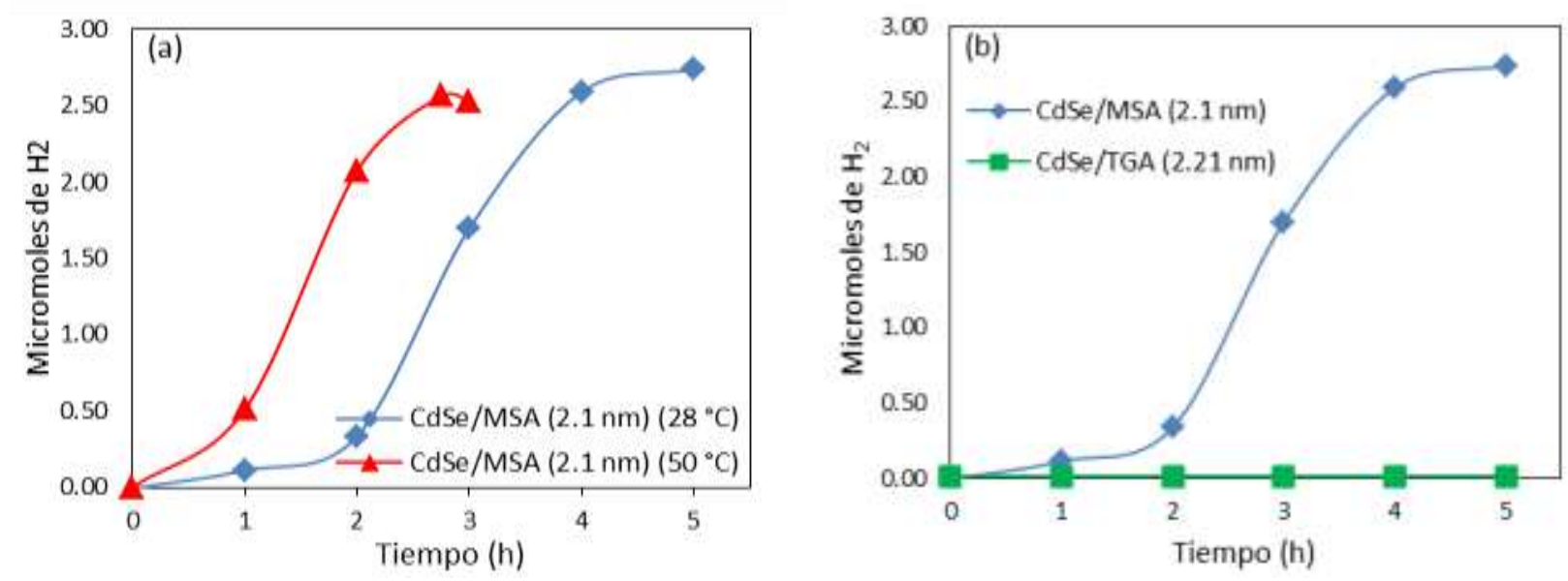

Fig. 10: (a) Evolución de hidrógeno a dos temperaturas $\left(28\right.$ y $\left.50^{\circ} \mathrm{C}\right)$ de la solución de PCs bajo irradiación (b) Evolución de hidrógeno de PCs con diferente capa envolvente (TGA o MSA)

En la Figura 10a se observa un comportamiento igual entre las dos curvas y tasas de evolución aproximadamente iguales $\left(1.192 \mu \mathrm{mol} \mathrm{H}_{2} / \mathrm{h}\right.$ en el tramo lineal de la curva de $\left.50^{\circ} \mathrm{C}\right)$. La diferencia entre las dos curvas radica en que el periodo de inactividad es casi nulo para $50^{\circ} \mathrm{C}$, es decir, la tasa de evolución constante de $\mathrm{H}_{2}$ empieza casi desde el principio. El aumento de la temperatura promueve la termalización de electrones y huecos, y por ende, una mayor cantidad de electrones atrapados en la banda de niveles superficiales por simple excitación térmica puede aumentar la tasa de reducción del $\mathrm{Cd}^{+2}$, acortando el periodo de inactividad inicial. 
Finalmente, para comparar el efecto de la composición superficial de los PCs, se realizó una prueba más con PCs de CdSe/TGA de diámetro promedio de $2.21 \mathrm{~nm}$. El resultado mostrado en la Figura 10b fue tasa de evolución cero, lo cual fue acorde con la caracterización previa realizada a los PCs con TGA.

\section{CONCLUSIONES}

De acuerdo con la investigación y los resultados analizados se pueden extraer las siguientes conclusiones principales:

i) EI MSA proporciona un mejor control de tamaño que el TGA durante la síntesis en iguales condiciones, aún después de la etapa de reflujo. Lo anterior se atribuye a la estructura del MSA que favorece una etapa de crecimiento limitada por difusión.

ii) El aumento de la concentración de TGA en la solución del precursor de Cd para aumentar el grado de pasivación superficial de los PCs durante la síntesis no indujo tal resultado. Los PCs con amplio ATMM requieren un proceso adicional de refinamiento de tamaño, por tanto los rendimientos productivos para obtener PCs de TGA con un tamaño específico fueron bajos.

iii) Los agentes estabilizantes son necesarios para mantener los PCs en suspensión en celdas fotoquímicas (PC/electrolito), sin embargo deben usarse moléculas estabilizantes que no afecten el contacto interfacial con donores o aceptores de electrones. La evaluación fotocatalítica realizada sugiere el uso de PCs con estrecho ATMM, bajo PL-QY, y con ligantes lineales de cadena corta dispuestos radialmente sobre la superficie, de manera que la especie reductora pueda moverse libremente a través de la capa envolvente. Por otra parte si se emplean PCs de CdSe/MSA, estos deben tener un PL-QY < 4\%, valor correspondiente a los PCs (2.1 nm) con la máxima tasa de evolución observada.

iv) Se evidenció la formación de $\mathrm{Cd}^{(0)}$ en los PCs al observar el oscurecimiento de la solución de naranja a café. El $\mathrm{Cd}^{(0)}$ actuó como un contra-electrodo para capturar electrones en la superficie, de forma similar al platino ( $\mathrm{Pt}$ ) depositado en la superficie de catalizadores en polvo. Lo anterior sugiere un pre-tratamiento de los PCs para mejorar las tasas de evolución de $\mathrm{H}_{2}$.

\section{AGRADECIMIENTOS}

Agradecimientos a la Universidad del Valle en el marco de convocatorias internas para la financiación de proyectos de investigación a nivel científico y tecnológico. Igualmente, gracias a la Universidad Libre de Bogotá por permitir el desarrollo de mediciones en fotocatálisis en el laboratorio de posgrado del programa de Ingeniería Ambiental. Triana agradece a Colciencias por la financiación de estudios de Doctorado.

\section{REFERENCIAS}

Dickerson B., Irving D., Herz E., Claus R., Spillman W., y Meissner K, Synthesis kinetics of CdSe quantum dots in trioctylphosphine oxide and in stearic acid, Applied Physics Letters: 86, 171915-1 - 171915-3 (2005)

Gaponik A., Talapin D., Rogach A., Hoppe K., Shevchenko E., Kornowski A., Eychmüller A y Weller H, ThiolCapping of CdTe Nanocrystals: An Alternative to Organometallic Synthetic Routes, J. Phys. Chem. B: 106, 7177-7185 (2002)

Gerischer H, Electrochim. Acta: 35, 1677-1699 (1990)

Holmes M., Towsend T y Osterloh F, Quantum confinement controlled photocatalytic water splitting by suspended CdSe nanocrystals, Chem. Commun: 48, 371-373 (2011)

Klayman D y Griffin T, Reaction of selenium with sodium borohydride in protic solvents. A facile method for the introduction of selenium into organic molecules, J. Amer. Chem. Soc: 95(1), 197-199 (1973)

Kniprath R., Rabe J., McLeskey J., Wang D y Kirstein S, Hybrid photovoltaic cells with II-VI quantum dot sensitizers fabricated by layer-by-layer deposition of water-soluble components, Thin solid films: 518, 295-298 (2009)

Mesa J., Machuca F., y Mueses M, Efecto de la Dispersión de Radiación sobre el Rendimiento Cuántico en la Degradación Fotocatalítica de Ácido Dicloroacético. Información tecnológica: 23(3), 33-42 (2012)

Mi W., Tian J., Tian W., Dai J., Wang X y Liu X, Temperature dependent synthesis and optical properties of CdSe quantum dots, Ceramics International: 38, 5575-5583 (2012) 
Morris T., y Zubkov T, Steric effects of carboxylic capping ligands on the growth of the CdSe quantum dots, Colloids and Surfaces A: Physicochem. Eng. Aspects, 443, 439- 449 (2014)

Perry R, Physical and chemical data, Perry's chemical engineers' handbook, 7th edition, McGraw-Hill, pp 142, New York, USA (1997)

Qu L., y Peng X, Control of Photoluminescence Properties of CdSe Nanocrystals in Growth, Journal of the American Chemical Society: 124(9), 2049-2055 (2002)

Suaterna N., Insignares C., Mueses M., y Camargo R, Fotodegradación Solar Heterogénea a Escala Piloto de 4-Clorofenol en un Reactor Cilindro Parabólico Compuesto (CPC). Información tecnológica: 23, (6), 13-24 (2012)

Wang Y., Lu J.,y Tong Z, Rapid synthesis of CdSe nanocrystals in aqueous solution at room temperature, Bull. Mater. Sci: 33(5), 543-546 (2010)

Wang Y., y Liu S, One-pot Synthesis of highly luminescent CdTe quantum dots using sodium tellurite as tellurium source in aqueous solution. J. Chil. Chem. Soc.: 57(2), 1109-1112 (2012)

Wang Y., Yang K., Pan H., Liu S y Zhou L, Synthesis of high-quality CdSe quantum dots in aqueous solution, Micro \& Nano Letters: 7(9), 889-891 (2012)

Yu W., Qu L., Guo W y Peng X, Experimental determination of the extinction coefficient of CdTe, CdSe, and CdS nanocrystals, Chem Mater: 15(14), 2854-2860 (2003)

Zhao J., Holmes M., y Osterloh F, Quantum Confinement Controls Photocatalysis: A Free Energy Analysis for Photocatalytic Proton Reduction at CdSe Nanocrystals, ACS Nano: 7(5), 4316-4325 (2013) 